\title{
Verrucous carcinoma of the nail bed in two patients with evidence of Human Papillomavirus infection
}

\author{
Ana Maria Calistru*, Paulo Santos, Paulo Morais, Filomena Azevedo \\ Department of Dermatology and Venereology, Hospital São João, Porto, Portugal
}

\begin{abstract}
Digital squamous cell carcinoma is relatively rare and may present a diagnostic challenge by mimicking benign conditions, such as verruca vulgaris. We describe two patients with longstanding verrucous lesions of the nail bed, which proved to be squamous cell carcinoma at the histopathology examination, with molecular and cytological evidence of HPV infection. Complete excision was performed. The cases highlight the necessity of a high index of suspicion in longstanding verrucous lesions of the digits, resistant to conventional anti-verruca vulgaris treatments.
\end{abstract}

Keywords: squamous cell carcinoma, nail bed, HPV

\section{Introduction}

Verrucous carcinoma is a well-differentiated squamous cell carcinoma with a distinct verrucous or exophytic appearance characterized by locally aggressive but minimal metastatic potential. The pathogenesis may be related to human papillomavirus (HPV) infection, chemical exposure or chronic inflammation. It involves oral or genital mucosa more than skin and the most frequent cutaneous location is the plantar surface.

\section{Case report}

We report a 47 year-old diabetic patient who presented with onycholysis and a verrucous plaque occupying the nail bed of the left thumb evolving for 8 years (Fig. 1).

Received: March 2014; Accepted after review: April 2014; Published: June 2014

${ }^{*}$ Corresponding author: Ana Maria Calistru, Department of Dermatology and Venereology, Hospital São João, Porto, Portugal. Email: anagruia@yahoo.com
The patient was previously unsuccessfully treated with phenol application and curettage. The biopsy of the lesion was consistent with verrucous carcinoma and HPV-16 was identified in the skin fragment by polymerase chain reaction test.

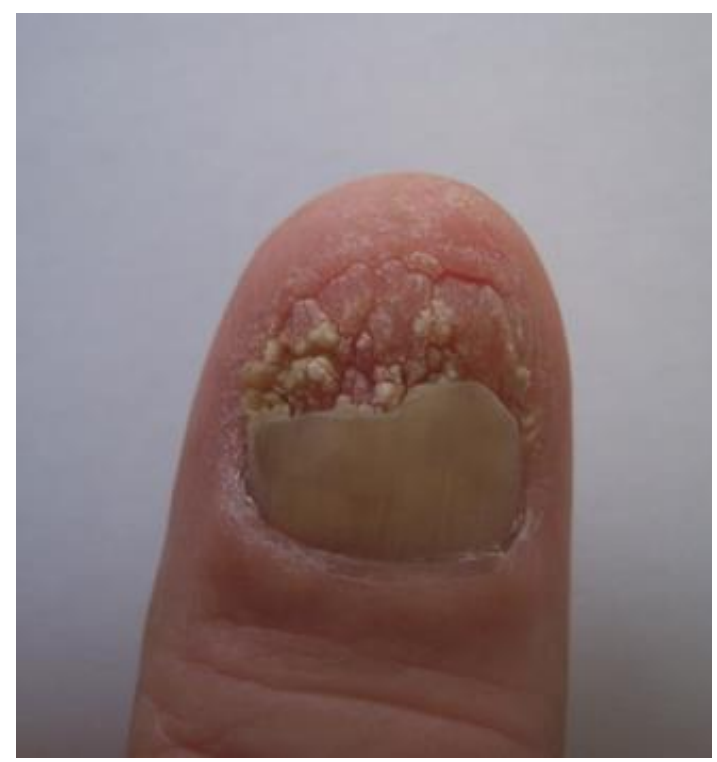

Fig.1. Case 1 - Verrucous carcinoma of the left thumb 
There were no palpable regional lymph nodes and the X-ray of the thumb did not show bone invasion. Complete blood cell count and basic biochemistry were irrelevant and the HIV serology was negative. An excision with a $4 \mathrm{~mm}$ safety margin and total surgical matricectomy were performed. There was no recurrence at 2 years of follow-up (Fig. 2).

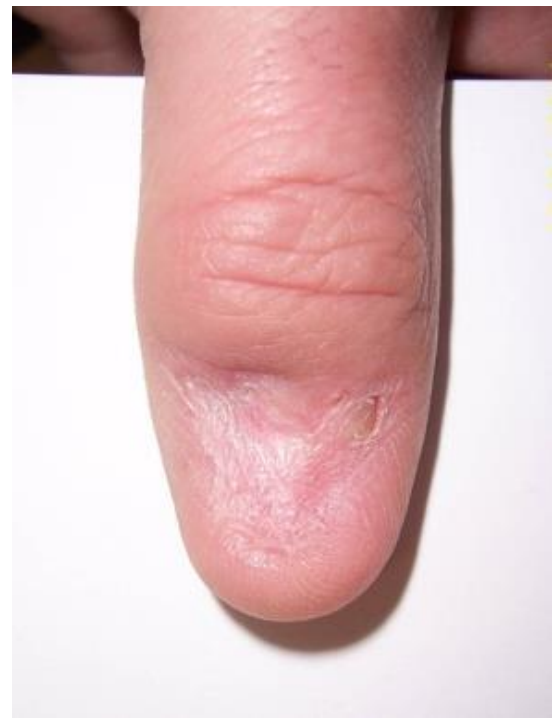

Fig. 2. Case 1 - Left thumb, 2 years after surgery

The second patient was a 49-year-old man with a warty nodule of the middle toe's nail bed evolving for 3 years (Fig. 3).

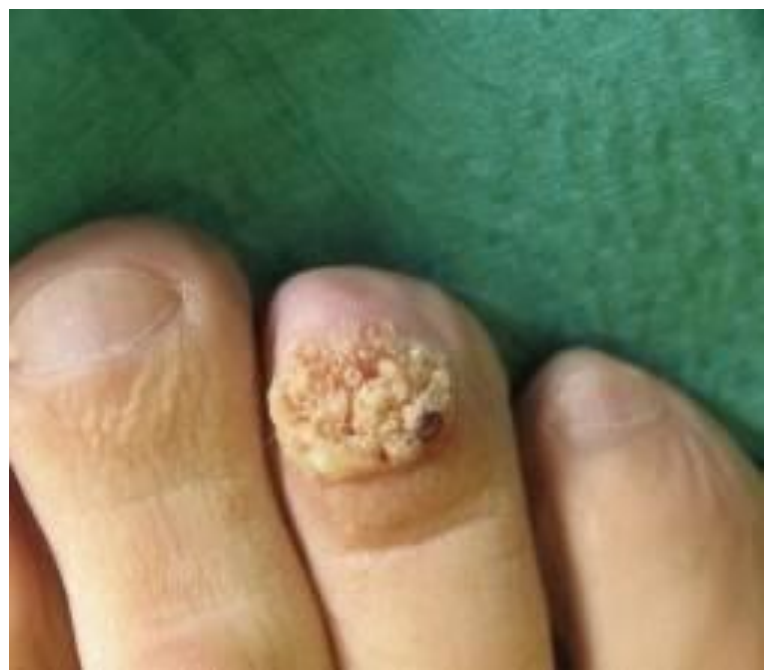

Fig. 3. Case 2 - verrucous carcinoma of the toe

He was HIV infected and had a CD4 count of $695 / \mathrm{mm}^{3}$. The biopsy revealed a verrucous squamous cell carcinoma invading the dermis and hypodermis and cytological changes of viral infection (Fig. 4).

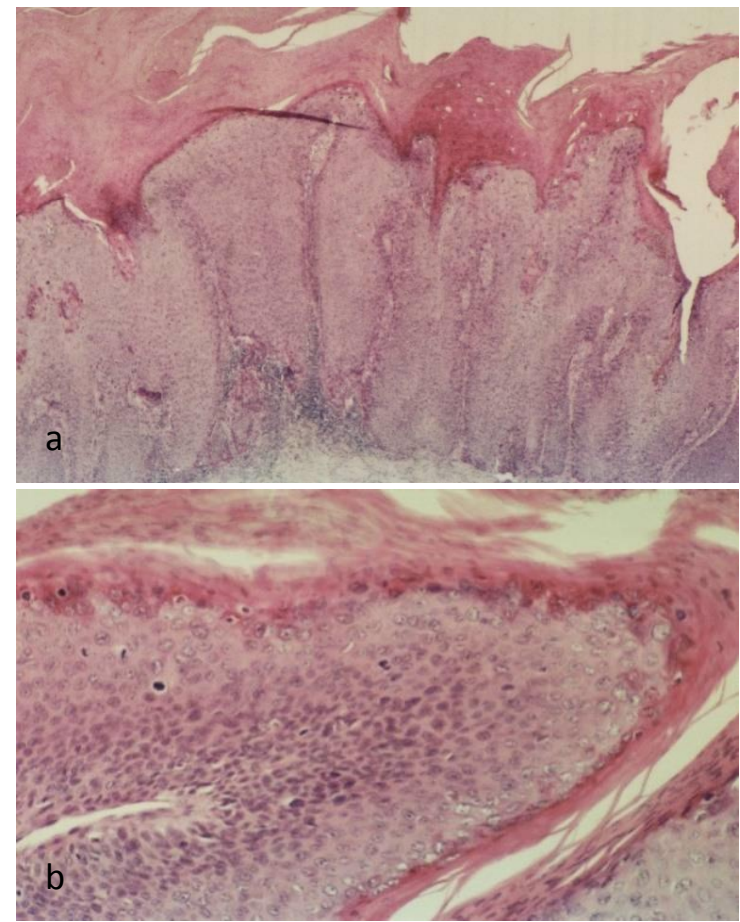

Fig. 4. Case 2 - invasive warty squamous cell carcinoma and cytological changes of viral infection (HE, x4 - a, x20-b)

Amputation of the distal phalange was performed with no recurrence after 12 months of follow-up (Fig. 5).

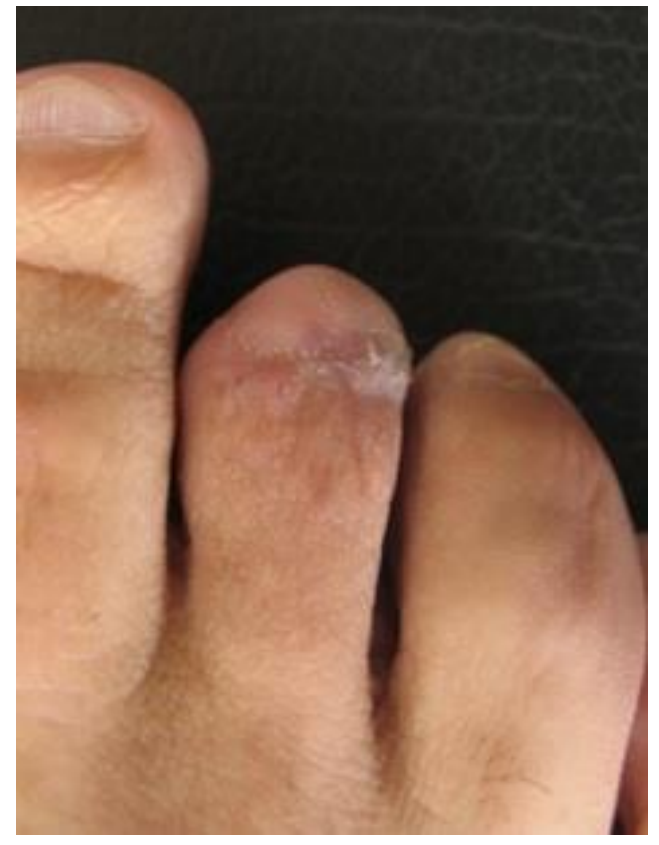

Fig. 5. Case $2-1$ year after surgery 


\section{Discussions}

Digital squamous cell carcinoma (SCC) is relatively rare and may present a diagnostic challenge by mimicking benign conditions, such as verruca vulgaris and chronic paronychia. Periungual and subungual warts caused by high-risk HPV subtypes pose a risk for malignant transformation [1]. The most commonly associated HPV type in digital SCC is HPV-16, although HPV-2, $-11,-18,-26,-31$, $34,-35,-56,-58$, and -73 also have been detected [2, 3]. Other risk factors are: exposure of the hands to ionizing radiation or carcinogenic chemicals, burns, genetic predisposition from specific genodermatoses and immunosuppression [2].

HPV-associated digital SCC may clinically present as single or multiple lesions, commonly as periungual verrucous plaque, subungual nodule and onycholysis, but longitudinal

\section{References}

1. Riddel C, Rashid R, Thomas V: Ungual and periungual human papillomavirus-associated squamous cell carcinoma: A review. J Am Acad Dermatol, 2011, 64(6):1147-53.

2. Hunt R, Hwa C, Tzu J, Patel R, Tyring SK, Stein $\mathrm{J}$ : Multiple human papillomavirus 16 associated digital squamous-cell carcinomas in an immunocompetent woman with prior human papillomavirus related genital carcinoma. Dermatol Online J, 2011, 17(10):20. melanonychia and lesions suggestive of paronychia and keratoacanthoma were also noted. Histologically, Bowen's disease or SCC in situ accounted for two thirds of the cancers, the remainder being predominantly invasive SCCs [4].

The rate of recurrence after surgery greatly exceeds that for cutaneous SCCs in general, being reported in $20-43 \%$, and may be caused by residual postsurgical HPV [2, 4]. The rate of metastasis, however, appears to be low (3\%) [4]. Given the high recurrence rate and proliferative activity of HPV-associated periungual SCCs, aggressive treatment and close follow-up of these tumors is mandatory [3]. A high index of suspicion is needed in order to make an early diagnosis.

These cases highlight the importance of the biopsy in the longstanding warty lesions of the digits in both immunocompromised and immunocompetent patients.

3. Kreuter A, Gambichler T, Pfister H, Wieland U: Diversity of human papillomavirus types in periungual squamous cell carcinoma. $\mathrm{Br} J$ Dermatol, 2009, 161(6):1262-9.

4. Alam M, Caldwell JB, Eliezri YD: Human papillomavirus-associated digital squamous cell carcinoma: literature review and report of 21 new cases. J Am Acad Dermatol, 2003, 48(3):385-93. 